\title{
Beam incidents - High particle rate tests of an LHCb/Velo silicon strip module
}

\author{
L. Eklund ${ }^{* a}$, K. Akiba ${ }^{b}$, O. Behrendt ${ }^{c}$, M. van Beuzekom ${ }^{b}$, J. Buytaert ${ }^{c}$, P. Collins ${ }^{c}$, M. \\ Ferro-Luzzi $^{c}$, K. Hennessy ${ }^{d}$, J. Imong ${ }^{e}$ \\ ${ }^{a}$ University of Glasgow, Glasgow, UK. \\ ${ }^{b}$ Nikhef, National Institute for Subatomic Physics, Amsterdam, Netherlands. \\ ${ }^{c}$ European Organisation for Nuclear Research (CERN), Geneva, Switzerland. \\ ${ }^{d}$ University of Liverpool, Liverpool, UK \\ ${ }^{e}$ H.H. Wills Physics Laboratory, University of Bristol, Bristol, United Kingdom. \\ E-mail: Lars.Eklundecern.ch
}

A silicon microstrip detector module from the $\mathrm{LHCb} / \mathrm{Velo}$ detector was exposed to proton rates in the range of $2 \times 10^{9}$ to $9 \times 10^{12}$ protons per pulse. The beam energy was $1.4 \mathrm{GeV}$ and the pulse lenght was $200 \mathrm{~ns}$, concentrated on a surface area of approximately $0.5 \mathrm{~cm}^{2}$. The sensor is of $n$-in$n$ type and AC-coupled to a front-end chip in $0.25 \mu \mathrm{m}$ CMOS technology. Both the active sensor area and the readout chips were exposed to successive beam pulses, at perpendicular impact. The module was powered with both low and high voltage, and read out during and between beam exposures. We report on the measurements of the backplane bias voltage collapse, of the leakage current, as well as noise and pedestal variations of the front-end readout. No degradation or damage was observed beyond those normally expected from the accumulated radiation dose.

VERTEX 2009 (18th workshop) - VERTEX 2009

September 13 - 182009

Veluwe, the Netherlands

\footnotetext{
* Speaker.
} 


\section{Introduction}

\subsection{The LHC beam environment}

The proton momentum and beam intensity at CERNs Large Hadron Collider (LHC [1]) will be such that the total energy stored in one beam, $360 \mathrm{MJ}$, is more than two orders of magnitude larger than the maximum beam energy stored in any previous collider, such as the Tevatron and HERA. Even during injection into the LHC, at $450 \mathrm{GeV}$, the energy stored in a single nominal batch of protons ( 288 bunches of each $1.15 \times 10^{11} \mathrm{p}$ ) will be $2.4 \mathrm{MJ}$, i.e. in excess of the maximum energy stored in a TEVATRON proton beam (1.5 MJ) or in a HERA proton beam (2 MJ).

Although a robust machine and experiment protection system has been developed [2] to cope with such extreme conditions, it is important to study the damage potential of possible, though unlikely, failure scenarios. In particular, beam failures at injection cannot be excluded. Compared to other LHC experiments, the LHCb detector [3] is particularly exposed to injection failures, because it is located in the region of injection of the anti-clockwise LHC beam. Furthermore, the LHCb experiment includes a dipole spectrometer magnet, compensated with three LHC magnets, all deviating the beams in the ring plane. Wrong magnet settings, in particular in the LHCb insertion region, could direct the beam into the LHCb detector [4]. Additionally, an incomplete or asynchronous beam kicker or wrong magnet settings in the transfer line could cause high particle rates in the $\mathrm{LHCb}$ experiment.

Beam failures can occur on different time scales. Slow, steady losses resulting from beam degradation on the time scale of seconds or minutes may damage the detectors, for instance by increased radiation dose. They do not necessarily require an automated beam abort since recovery of good beam conditions may be attempted. On the contrary, faster losses require rapid, automated reaction. For example, beam losses due to a tripping magnet will generally develop on the time scale of several turns ${ }^{1}$ (for warm magnets) to several milliseconds (for superconducting magnets). Ultra-fast losses, on the time scale of one turn or less, are tackled by passive protection. Such losses are due to e.g. an injection failure or a beam extraction failure.

To mitigate the risk for injection failures, a number of movable and fixed absorbers are placed upstream of the $\mathrm{LHCb}$ experiment [5]. Furthermore, machine protection interlocks guarantee that injection into an empty LHC ring starts with a bunch intensity not in excess of the probe beam limit $^{2}$, in order to probe at once the settings of all static beam-steering elements of the machine and transfer line and to detect unexpected aperture limitations. Once circulating beam is established, injection of high intensity batches may proceed.

In the current configuration of the LHC machine protection system, and assuming specific failure scenarios, a $450 \mathrm{GeV}$ proton bunch of up to $10^{10} \mathrm{p}$ could be mis-injected and directed through the innermost detectors of an LHC experiment. The beam size at the experiment in such a case would be of the order of $\sim 0.3 \mathrm{~mm}$ RMS in the two transverse directions. Therefore, this represents potentially a particle density of up to about $5 \times 10^{12} \mathrm{p} / \mathrm{cm}^{2}$.

\footnotetext{
${ }^{1}$ One LHC turn is about $89 \mu \mathrm{s}$.

${ }^{2}$ The probe beam limit is initially set to $10^{10} \mathrm{p}$ and could be configured to a maximum of $10^{11} \mathrm{p}$ under special conditions
} 


\subsection{Background and motivation}

The LHCb Vertex Locator (VELO [6]) is a particularly exposed detector due to its vicinity to the beam axis. It is composed of 42 silicon strip modules located in a secondary vacuum, divided in two halves that can be retracted sideways by $30 \mathrm{~mm}$ during LHC filling. The 21 Velo modules of each half are enclosed in a thin-walled Aluminium box $(\sim 300 \mu \mathrm{m})$ that separates the beam vacuum from the detector vacuum. In nominal physics operation the silicon detectors will be precisely positioned around the colliding beams and the Aluminium box will approach the beams to a mere $5 \mathrm{~mm}$ distance (the silicon edges reaching a radial distance of about $7 \mathrm{~mm}$ ).

The risk of damage for silicon detectors depends on the detailed design of the sensors, frontend hybrid and read-out electronics. The Velo strip sensors are $n$-in- $n$ and have an area of approximately $27 \mathrm{~cm}^{2}$. The 2048 strips are AC coupled and connected to the read-out chip via wire bonds. Each module has two sensors mounted back to back, one measuring the radial coordinate $(R$-sensor) and one measuring a pseudo-azimuthal coordinate ( $\Phi$-sensor). The strip-side of the sensor is at ground potential and a negative high voltage is applied to the back plane via a two-stage $\mathrm{RC}$ filter. The connection to the back plane is done via conductive glue to pads located on the front-end hybrid. An equivalent circuit relevant to the processes involved in the high rate tests is shown in Figure 1. The sensors are read out by the Beetle [7] front-end chip that is made in a $0.25 \mu \mathrm{m}$ CMOS process.

All vertex detectors and innermost trackers at LHC experiments are based on silicon technology. Hence their ability to survive beam incidents is a common problem. Previous studies with lasers [8][9] on biased sensors have shown that large voltage gradients are generated and may cause damage if focused on a sufficiently small $\mathrm{area}^{3}$. Studies with proton beams [10][11] reached intensities of $10^{11}$ protons over approximately $20 \mathrm{~cm}^{2}$ and did not cause any damage.

Beam-induced damage for silicon detectors may have different causes, among which: direct energy deposition of the beam, radiation damage and beam induced transients originating from the energy stored in the system. In addition, large ionization can create Single Event Effects (SEE) in the front-end electronics.

- Energy deposition: A rough estimate ${ }^{4}$, neglecting particle showering in the silicon, suggests that an instantaneous rate of $\sim 10^{13}$ protons $/ \mathrm{cm}^{2}$ will only increase the local temperature by a few degrees. Given the lightness of silicon vertex detectors and the assumed beam failure scenarios, heat-induced damage to silicon seems unlikely. Thermal shock effects have not been considered in details for our detector.

- Radiation damage: Silicon detectors at LHC experiments are designed to survive fluences of several $10^{14} \mathrm{n}_{\mathrm{eq}} / \mathrm{cm}^{2}$, corresponding to an absorbed dose of about $10 \mathrm{MRad}^{5}$. Provided that beam incidents are rare and uniformly spread, they will not contribute significantly to the accumulated dose.

- Beam induced transients: In addition to the energy deposited by the beam, there is energy stored in the sensor and front-end hybrid if sensor bias is applied. In particular the charge

\footnotetext{
${ }^{3}$ One strip in the case study presented in [8]

${ }^{4} \Delta T \approx\left(1.66 \mathrm{MeV} \mathrm{cm}^{2} / \mathrm{g}\right) \cdot R / C_{p}=3.8 \mathrm{~K}$, with a specific heat of silicon $C_{p} \approx 0.7 \mathrm{Jg}^{-1} \mathrm{~K}^{-1}$.

${ }^{5}$ Equivalent non-ionizing energy loss: $1 \mathrm{n}_{\mathrm{eq}}=1 \mathrm{MeV}$ neutron equivalent displacement damage in silicon.
} 


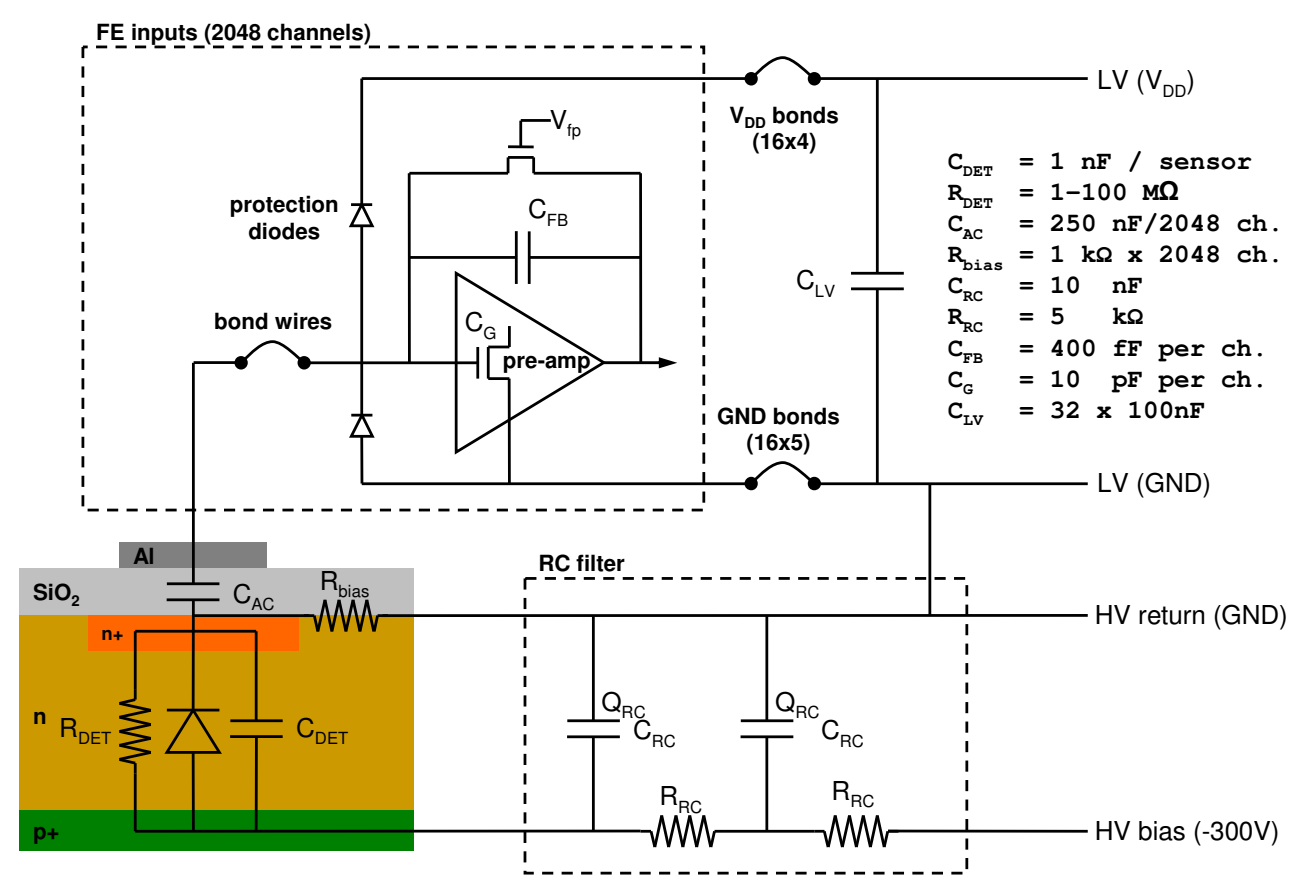

Figure 1: A simplified electrical model of the sensor, front-end hybrid and read-out ASICs. The model includes the components that are relevant to the high rate tests.

stored in the capacitors $C_{D E T}$ and $C_{R C}$ in Figure 1 can generate large instantaneous currents when discharged by large ionization. These currents could cause damage by locally creating large voltage spikes or current densities.

- Single Event Effects: Large localised energy depositions can create destructive (e.g. Single Event Latch-up) or transient (e.g. Single Event Upsets) effects in electronic circuits. The ionization density from protons is not sufficient to cause such effects, they can only be created by displacement of nuclei from the crystal lattice. The front-end ASICs used in the LHC experiments are designed to minimise these effects.

\section{Measurements}

\subsection{Beam line}

The high rate tests were performed in a beam measurement line of the PS Booster at CERN, Geneva. The PS Booster can deliver intensities from $2 \times 10^{9}$ to $9 \times 10^{12}$ protons per spill, where the lower limit is determined by the sensitivity limit of the beam instrumentation. The length of one bunch is approximately $200 \mathrm{~ns}$ and was focused down to a size of $0.5 \mathrm{~cm}^{2}$. As a comparison, 
the beam structure in the LHC is $10^{11}$ protons per $0.4 \mathrm{~ns}$ bunch, with 288 bunches in one injection train.

The Velo module was mounted on a movable stage close to the dump in the beam line and could be inserted and extracted from the beam remotely. A luminescent screen was mounted in front of the module that could be inserted independently of the module. The screen has a visible grid hence the location of the beam on the module could be determined. The beam size and position can be adjusted by the operators and viewed on the luminescent screen via a beam line camera [12]. Once the beam was set up using the screen and the set-up was prepared for a measurement, the module was moved in to receive one bunch of protons.

\subsection{Experimental set-up}

In order to monitor the performance of the silicon strip module before and after each shot with the proton beam, it has to be powered, configured and read-out remotely. This was achieved via bench top power supplies and a stripped down version of the LHCb/Velo control and read-out system. All power supplies and read-out electronics was installed remotely ( $\sim 50$ meters $)$ in the booster control room. A high voltage supply was used to sensor bias during the beam shots and to make I/V measurements between them. Hence noise, gain and pedestals could be measured in-situ as a function of bias voltage.

The module accumulated a non-negligible radiation dose due to the proximity to the beam dump. The radiation monitoring devices [13] show that the accumulated radiation fluence from the background was approximately $10^{13} \mathrm{n}_{\mathrm{eq}} / \mathrm{cm}^{2}$. Hence cooling was necessary not only to remove the heat from the front-end electronics but also to reduce the radiation induced leakage current in the sensor.

The PS Booster provided a beam spill signal that was used to trigger the read-out of 15 consecutive events at the arrival of the bunch, sampled at $25 \mathrm{~ns}$ interval. These events were analysed to investigate the behaviour of the front-end during the shot. Furthermore, a digital oscilloscope was connected across the RC filter capacitance $\left(C_{R C}\right.$ in Figure 1$)$ on the front-end hybrid. The differentially measured voltage correspond to the voltage of the back-plane w.r.t. hybrid ground. The beam spill signal triggered the read-out of $200 \mathrm{k}$ samples with $1 \mathrm{~ns}$ interval. The results of these measurements are presented in Section 3.1.

\subsection{Measurement programme}

A measurement programme was performed in increasing steps of beam intensity and high voltage configuration. The aim was to immediately spot any signs of damage in-situ and to determine the damage limit of the module. The measurements were done at five intensities between $2 \times 10^{9}$ and $9 \times 10^{12}$ in four different configurations of the low voltage (LV) and sensor bias (HV). A summary of the number of beam shots in each configuration is shown in Table 1.

The beam was also directed at four different front-end ASICs, at three different intensities to probe the sensitivity to Single Event Effects. The number of shots with low voltage on and off are also shown in Table 1. 
Table 1:

Summary table of the number of beam shots on the sensor and the front-end ASICs at the different beam intensities and module configurations. Intensities are given in protons per $200 \mathrm{~ns}$ bunch.

\begin{tabular}{|c|cccc|cc|}
\hline \multirow{3}{*}{ Intensity } & \multicolumn{5}{|c|}{ Shots on the sensor } & \multicolumn{2}{c|}{ the ASIC } \\
& LV off & LV on & LV on & LV on & LV off & LV on \\
& HV 0 V & HV 0 V & HV 150 V & HV 300 V & & \\
\hline $2 \times 10^{9}$ & 1 & 2 & 33 & 2 & 13 & 8 \\
$2 \times 10^{10}$ & 1 & 1 & 1 & 1 & 15 & 17 \\
$2 \times 10^{11}$ & 1 & 1 & 1 & 1 & 3 & - \\
$2 \times 10^{12}$ & 1 & 1 & 1 & 1 & - & - \\
$9 \times 10^{12}$ & 2 & 2 & 5 & 5 & - & - \\
\hline
\end{tabular}

\section{Results}

\subsection{In-situ measurements}

Figure 2 shows the voltage difference between the the sensor back-plane and hybrid ground, measured across the capacitor $C_{R C}$ in Figure 1. The plots show data from shots at the minimum and maximum beam intensity at $150 \mathrm{~V}$ sensor bias. Two main features are visible in the plots. First a very fast collapse of the back-plane voltage ( $\sim 50 \mathrm{~ns})$ and then a slow recovery $(\sim 20 \mu \mathrm{s})$. The fast and nearly complete collapse of the bias voltage is due to the fact that even at the lowest beam intensity $\left(2 \times 10^{9}\right.$ protons) the total charge deposited by the beam ${ }^{6}$ is larger than the charge stored in the capacitors $C_{R C}$ and $C_{D E T}{ }^{7}$. Hence a major beam incident will drain all the charge from the filter capacitors on the hybrid, and depositing more charge in the sensor than is available in these capacitors will not increase the magnitude of the electrical transients. The observed gradients of the rising edges of the back plane voltage collapse were between 2 and $6 \mathrm{GV} / \mathrm{s}$. The larger values were in general seen for higher bunch intensities and the shot to shot variation is probably due to the particular shape of each proton bunch.

The slow return of the voltage comes from the re-charge of the capacitors $C_{R C}$ and $C_{D E T}$ by the HV bias supply. The induced transients from this process are much smaller than for the initial discharge because of the longer time constants involved.

The analysis of the the of 15 consecutive events triggered by the beam spill signal showed a consistent picture with what was observed by the oscilloscope measurements. A beam spot could be observed for some shots where the number of protons arriving during the first trigger of the arriving bunch was sufficiently low. The following samples showed saturated ADC values for all strip channels induced by the extremely fast collapse of the back plane voltage. For even later triggers the communication with the module was lost due to the large ground bounces induced by the beam. The oscilloscope measurements showed that the hybrid ground moved more than $30 \mathrm{~V}$ at the arrival of the beam.

\footnotetext{
${ }^{6} Q=2 \times 10^{9}$ protons $\cdot 3.8 \mathrm{fC} /$ proton $=7.6 \mu \mathrm{C}$

${ }^{7} Q=150 \mathrm{~V} \cdot 10 \mathrm{nF}=1.5 \mu \mathrm{C}$
} 

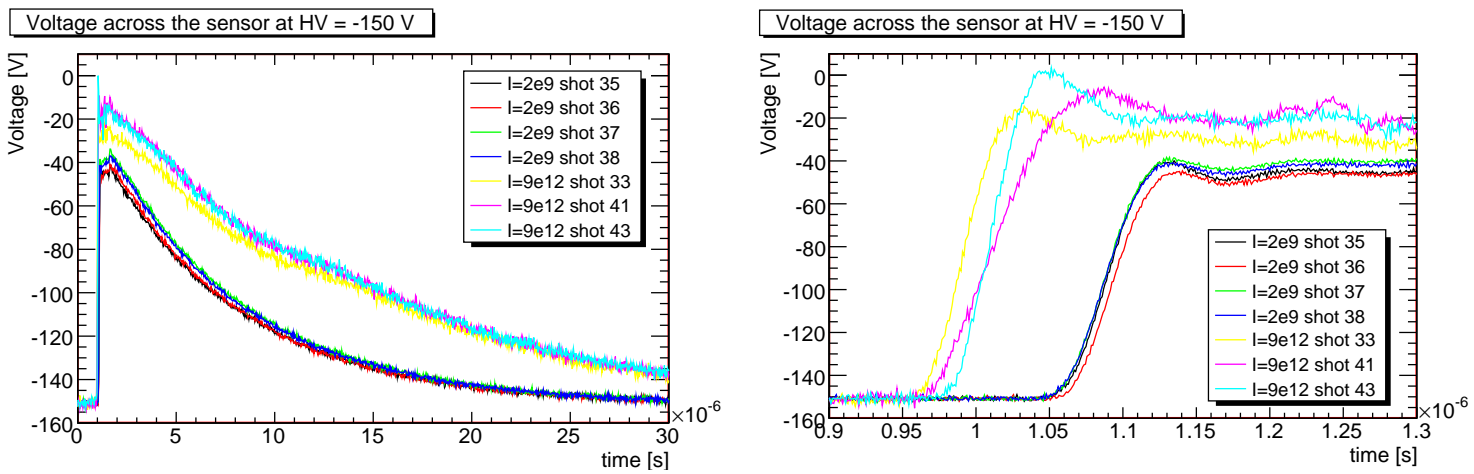

Figure 2: The voltage between the sensor back-plane and the hybrid ground versus time after the arrival of the beam spill signal. Each curve represents one beam spill. The plot on the left shows the fast collapse and re-charge of the bias voltage. The plot on the right shows a close-up of the first $400 \mathrm{~ns}$.
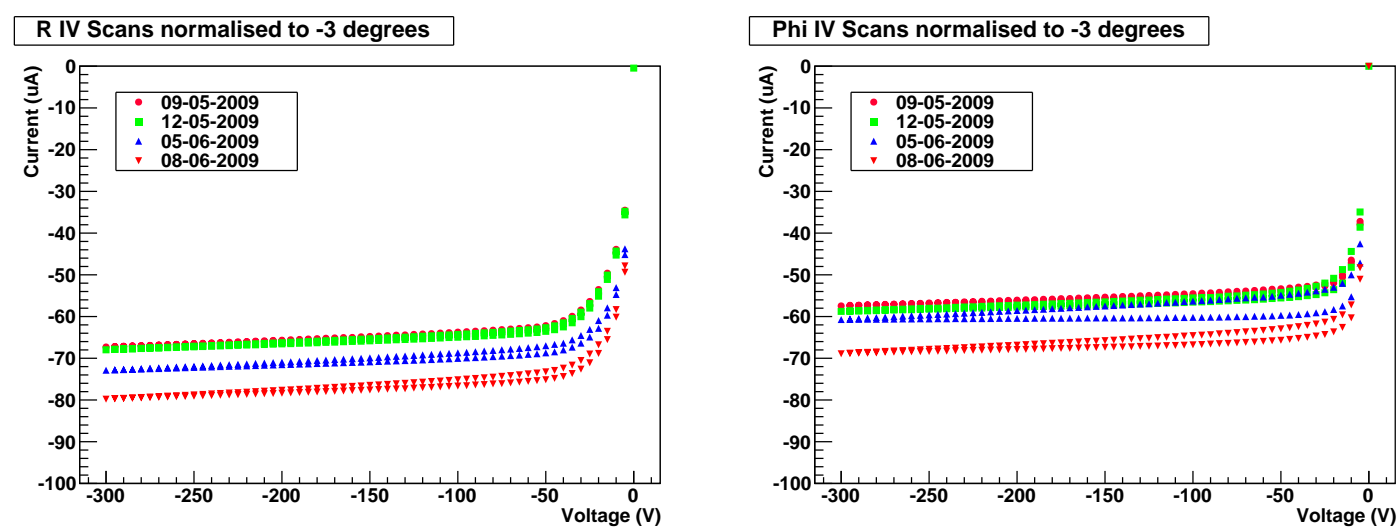

Figure 3: A series of $I / V$ curves measured in-situ during the irradiation programme for the two sensors on the module. The I/V curves for the sensor measuring the radial coordinate $(R$-sensor) are shown on the left and for the sensor measuring the azimuthal coordinate $(\Phi$-sensor) on the right. The small increase in leakage current over time is compatible with the measured accumulated radiation damage from the background.

The noise, pedestals and gains were measured at different bias voltages between the beam shots and they remained unchanged during the whole irradiation programme. Figure 3 shows a series of I/V curves taken at different times during the programme. No drastic change in leakage current was observed, only a small gradual increase. This is believed to be due to the accumulated radiation dose from the back-splash of particles from the beam dump.

The 56 shots on the front-end chips did not induce any destructive single event effects. All chips could be re-configured and operated normally afterwards.

\subsection{Laboratory measurements}

The measurements of noise, pedestals and gain were repeated in laboratory measurements after that the module had been dismounted from the beam. These measurement did not show any indications of damage, neither of the sensor nor of the front-end chip. Similarly the I/V measurements 
made in the laboratory confirmed the observations from the beam line.

\section{Discussion and conclusions}

A silicon detector module from LHCb Velo was exposed to beam shots of intensities up to $9 \times 10^{12}$ protons with up to $300 \mathrm{~V}$ sensor bias. No damage was observed beyond what is expected from the accumulated dose, and the detector module was fully functional. The expected damage would, as discussed in Section 1.2, most likely come from current or voltage transients induced by the beam. The damage caused by these transients include pin-holes through the AC coupling oxide, fusing of bond wires and destruction of the front-end amplifier. The size and consequences of these transients are reduced by the following key points.

- The energy available for fast transients come from the beam itself and the RC filter capacitor located closest to the sensor $\left(C_{R C}\right)$. This capacitor is of relatively moderate size $(10 \mathrm{nF})$ and will be drained completely in major incidents, reducing the bias voltage to zero.

- The highest current concentration is from this capacitor to the back plane of the sensor. This connection is made with conductive glue in several spots and hence is both robust and has low impedance.

- In these tests and in any likely beam incident, the beam covers many ( $>30)$ strips. Hence the initial current will be spread over several strips and when $C_{R C}$ starts to drain the back plane voltage is reduced and the sensor reacts as one unit, spreading the currents across all channels. Even if the voltage gradients in Figure 2 indicate an instantaneous current of $\sim 50 \mathrm{~A}$, it translates into a moderate current per channel.

- The AC coupling oxide can be damaged if a too large voltage occurs between the implant and the aluminium strip. The maximum possible voltage in this scenario is if the full charge of $C_{R C}$ is transferred to $C_{A C}$ without any current escaping through the bias resistors or punchthrough protection structures. This voltage is reduced by the ratio between $C_{R C}$ and $C_{A C}{ }^{8}$ shown in Figure 1.

- The Beetle chip has protection diodes, indicated in Figure 1, to clamp the voltage of the strip channel inputs. These provide a sufficiently low impedance path to ground to keep the voltage excursions below the damage limit.

- There is a sufficient number of ground bonds to drain the current required through the protection diodes.

- The triple redundancy designed in key logic circuits worked very well to reduce the sensitivity to Single Event upsets. Also, the technology proved to be resistant against latch-up and other SEE.

${ }^{8} V_{A C}=V_{\text {bias }} \cdot C_{R C} / C_{A C} \approx V_{\text {bias }} / 25$ 


\section{Acknowledgements}

We would like to express our gratitude to Raphael Dumps (CERN/PH-DT) for designing and building the high rate test setup, to the CERN/BE-BI group, in particular Stephane Burger, for providing the beam spot images, to the CERN/BE-OP group for their support, in particular David Allen for tuning the beams, Fabrice Chapuis for organising the beam ejection signal and Klaus Hanke for accommodating our experiment into the PS Booster machine development schedule. Finally, we would like to thank Maurice Glaser (CERN/PH-DT) for providing the RADMON sensor and associated data analysis.

\section{References}

[1] O. Brüning, et al., editors, "LHC Design Report v.1: the LHC Main Ring”, CERN-2004-003-V-1a.

[2] R. Schmidt et al., "Protection of the CERN Large Hadron Collider", New J. of Phys. 8 (2006) 290.

[3] A. A. Alves et al.[LHCb Collaboration], "The LHCb Detector at the LHC," JINST 3 (2008) S08005.

[4] R.B. Appleby, "LHCb Injected Beam Accidents", LHC Project Report 1174.

[5] V. Kain, "What is require to safely fill the LHC", 3rd LHC Project Workshop, Divonne-les-Bains, France, Jan 2006, CERN-AB-2006-014

[6] "LHCb VELO: Technical Design Report” Barbosa-Marinho, P R et al., CERN-LHCC-2001-0011

[7] S. Loechner, M. Schmelling, LHCb Note LHCb-2005-105

[8] K. Hara, T. Kuwano, G. Moorhead, Y. Ikegami, T. Kohriki, S. Terada and Y. Unno "Beam splash effects on ATLAS silicon microstrip detectors evaluated using 1-w Nd:YAG laser", Nucl.Instrum. Methods Phys. Res. A541 (2005) 15.

[9] T. Dubbs, M. Harms, H. F. W. Sadrozinski, A. Seiden and M. Wilson, "Voltages on silicon microstrip detectors in high radiation fields," IEEE Trans. Nucl. Sci. 47 (2000) 1902.

[10] A. Andreazza, K. Einsweiler, C. Gemme, L. Rossi, P. Sicho, "Effect of accidental beam losses on the ATLAS pixel detector", Nucl. Instrum. Methods Phys. Res. A565 (2006) 50.

[11] M. Fahrer, G. Dirkes, F. Hartmann, S. Heier, A. Macpherson, Th. Müller, Th. Weiler, "Beam-loss-induced electrical stress test on CMS Silicon Strip Modules", Nucl. Instrum. Methods Phys.Res. A518 (2004) 328.

[12] E.Bravin, S.Burger, G. Ferioli, G.J. Focker, A. Guerrero, R. Maccaferri, "A new TV beam observation system for CERN" 7th European Workshop on Beam Diagnostics and Instrumentation for Particle Accelerators (DIPAC 2005), Lyon, France, 6 - 8 Jun 2005, pp. 212.

[13] F. Ravotti, M. Glaser, A.B. Rosenfeld, M. Lerch, A.G. Holmes-Siedle, G. Sarrabayrouse, "Radiation Monitoring in Mixed Environments at CERN: From the IRRAD6 Facility to the LHC Experiments" IEEE Transactions on Nuclear Science, Vol. 54, Issue 4, Aug. 2007, Pages: 1170 - 1177. 